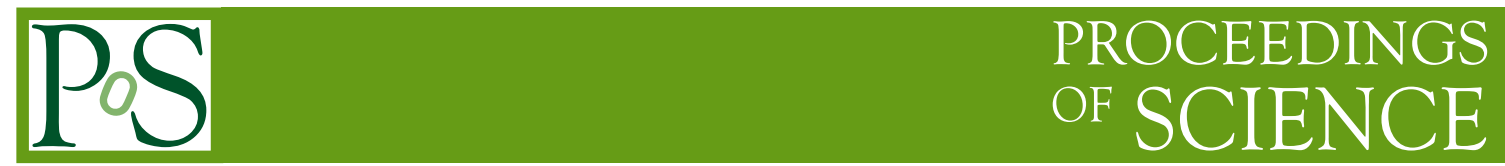

\title{
Advances in QCD Predictions
}

\section{Thomas Gehrmann*}

Department of Physics, University of Zurich, $\mathrm{CH}-8057$ Zurich, Switzerland

E-mail: thomas.gehrmann@uzh.ch

Precision calculations in QCD are crucial to ensure the optimal exploitation of LHC data in terms of Standard Model precision measurements and new physics searches. This talk reviews the recent progress in QCD predictions for collider physics, and highlights important developments and future challenges.

XXIV International Workshop on Deep-Inelastic Scattering and Related Subjects

11-15 April, 2016

DESY Hamburg, Germany

${ }^{*}$ Speaker. 


\section{Introduction}

The ATLAS and CMS experiments are performing precise measurements of a broad range of Standard Model processes, up to high multiplicities of final state objects. These data contain important information on the basic parameters of particle physics. Precision measurements of lowmultiplicity benchmark observables can be used to search for indirect evidence of new physics, and high-multiplicity final states are backgrounds in direct searches for new unstable particles. A close interplay between theory and experiment is required to interpret and analyse these data in order to ensure their optimal physics exploitation.

The theoretical description of LHC observables relies on two different approaches: perturbative calculations and parton showers. Perturbative calculations are based on an expansion of the relevant scattering matrix elements in powers of the coupling constant, at leading order (LO), nextto-leading order (NLO) and so on. They correctly describe hard radiation patterns, do however fail to account for full event characteristics like jet substructures or particle multiplicities, which are determined by soft dynamics. Resummation of large logarithmic corrections helps to extend the scope of perturbative calculations. The perturbative approach has a well-determined theoretical accuracy, which can be further improved by computing higher order corrections. The leading logarithmic behaviour of QCD reactions forms the basis of the parton shower approach, which is at the basis of all Monte Carlo programs that allow for the simulation of full event characteristics, including empirical models for hadronization. In recent years, more and more elements of the perturbative description have been incorporated into the Monte Carlo programs, increasing their quantitative accuracy and reliability.

\section{Multi-particle final states}

A reliable description of multi-particle final states is crucial for searches of beyond Standard Model effects and for the study of standard model processes involving heavy instable particles. To obtain a quantitative description of rates and shapes of distributions, and to account properly for hard radiation and jet algorithm dependence, NLO accuracy appears to be mandatory. Enormous progress (NLO revolution) has been made in the past years in the automation of NLO corrections to processes of arbitrary multiplicity, largely enabled by advances in techniques for the computation of loop amplitudes as well as by a standardisation of interfaces between different contributions (Born level, real corrections, virtual corrections, subtraction terms) through the Binoth Les Houches accord (BLHA, [1]). Several codes are now available for the automated computation of one-loop amplitudes: BlackHat [2], GoSam [3], OpenLoops [4], NJet [5], MadLoop [6], CutTools [7]. Through the BLHA interface, they can be readily combined with standard Monte Carlo simulation programs (HERWIG [8], SHERPA [9], MadGraph/aMC@NLO [10]) that also supply the full infrastructure (phase space, amplitudes and subtraction terms, analysis routines) for the prediction of physical observables and allow a matching of the predictions onto parton shower models. These implementations complement the dedicated libraries of NLO processes MCFM [11] and VBFNLO [12]. This technical process has led to an impressive list of newly available high-multiplicity NLO calculations, including multi-jet production [13], gauge boson production with up to five jets [14], gauge boson pairs with up to two jets [15, 16], triple gauge boson production [17], Higgs boson 


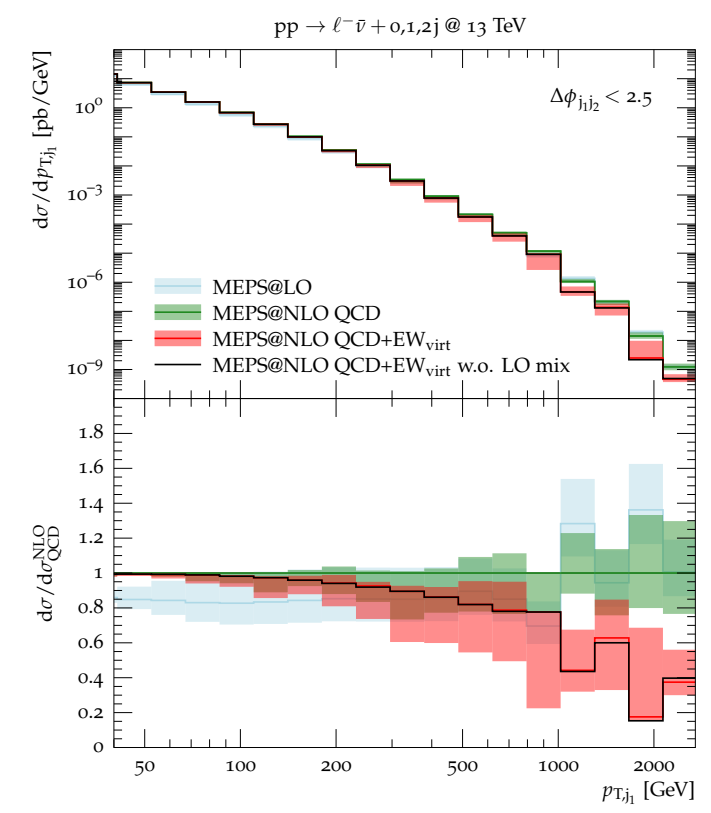

Figure 1: Matched NLO predictions for $W+$ multijet production, including electroweak corrections. Figure from [26].

production with up to three jets $[18,19]$ and many more, all with broad implications for precision phenomenology. All these processes are computationally expensive, and new ideas are needed to facilitate the incorporation of the results into the experimental analysis. The distribution of NLO event-ntuples [20] on which experimental analyses can be carried out emerges as a very promising format.

NLO calculations for a specific final state multiplicity can be routinely combined with parton showers using the MC@NLO [21] or POWHEG [22] methods. To obtain fully NLO-accurate event samples, one needs to merge NLO calculations of different multiplicities with each other. Three methods have been developed for this aim: MENLOPS [23], MiNLO [24] and UNLOPS [25] and are now starting to be used routinely in precision calculations of multi-particle final states. A major extension of automated NLO calculations is the inclusion of electroweak corrections, which was accomplished recently in OpenLoops [26] and MadGraph/aMC@NLO [27]. Figure 1 shows the matched prediction for $W+$ multijet production [26], including NLO QCD and electroweak effects.

The parton shower resums all leading logarithmic (LL) corrections from infrared emissions, and correctly describes many subleading logarithmic effects. To go beyond this level, dedicated resummations of specific logarithms (e.g. threshold, transverse momentum, jet size) are performed for specific observables. In the recent past, generic frameworks were developed for N3LL resummation of threshold logarithms [28] and for NNLL resummation of transverse momentum [29], with first steps [30] towards N3LL accuracy for this type of logarithms. The resummation of jet size logarithms has been developed [31] and finds applications especially in the optimization of jet veto techniques in Higgs production [32], see Figure 2.

A systematic framework for logarithmic resummations is provided by soft-collinear effective theory (SCET). In this framework, an effective theory for jet processes [33] and a generic NNLL 

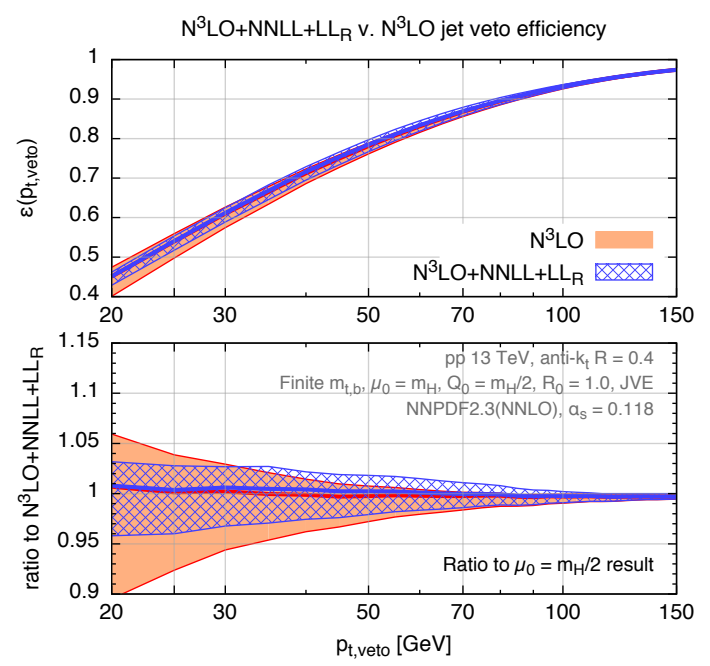

Figure 2: Jet veto efficiency in fixed order and including resummation. Figure from [32].

endpoint resummation for event shapes [34] were developed. The $\mathrm{N}$-jettiness event shape variable [36] was used to develop a new jet algorithm Xcone [35] and to study multi-scale hierarchies between jets [37]. A perturbative expansion of the SCET ingredients (soft functions [38] and beam functions [39]) allows to systematically improve the logarithmic accuracy of SCET resummation.

With the combined advances in NLO calculations, their incorporation into widely used parton shower event generator programs, and with progress in analytical resummation techniques, elementary particle theory enables quantitatively reliable predictions for a large spectrum of LHC observables, thereby preparing the optimal exploitation of LHC data from the current run.

\section{Benchmark observables}

The relative accuracy or the order of ten per cent that can be achieved with NLO calculations is sufficient to match the experimental precision for most multi-particle production processes. Several basic low-multiplicity processes are however measured to a much higher precision, and can be used for the determination of fundamental parameters. To interpret these benchmark observables, corrections beyond NLO become mandatory. Substantial advances in computational methods for next-to-next-to-leading order (NNLO) calculations have enabled new results for a broad spectrum of processes during the past two years.

The calculation of NNLO corrections to an $n$-particle process requires three types of contributions: two-loop virtual corrections to the $n$-particle amplitude, one-loop virtual corrections to the $(n+1)$-particle amplitude and the tree-level $(n+2)$-particle amplitude. Two-loop virtual amplitudes are available for almost all $(2 \rightarrow 2)$ processes of phenomenological interest, and one-loop and tree-level contributions appear already as part of higher-multiplicity NLO calculations, where they can be generated with automated tools. All three types of contributions are separately infrared divergent, only their sum is finite. The extraction of the infrared divergencies from the real radiation processes at tree level and one loop has been a major challenge, that was overcome only recently 


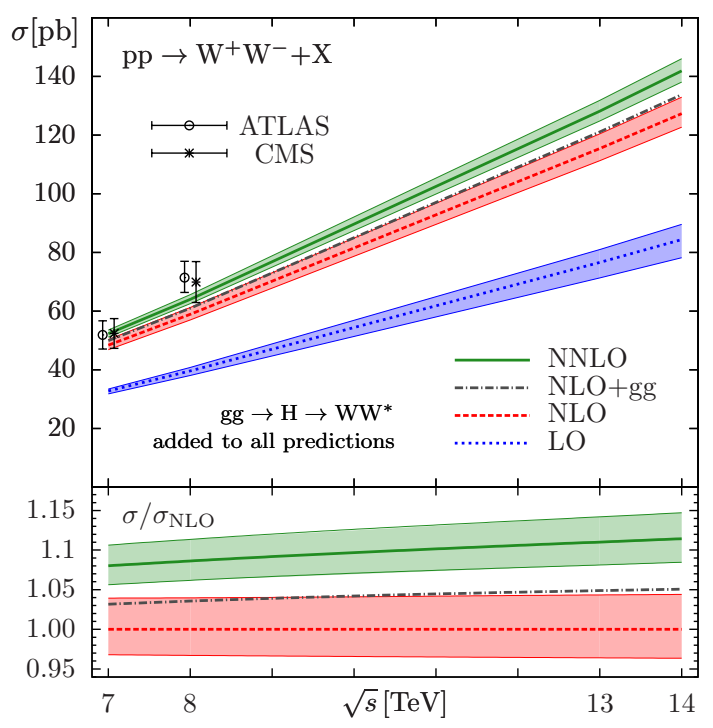

Figure 3: Inclusive cross section for $W^{+} W^{-}$production at NNLO. Figure from [50].

with the development of several new methods. Complementing and extending the scope of the previously available methods of sector decomposition [40] and $q_{T}$-subtraction [41], the methods of sector-improved residue subtraction [42], antenna subtraction [43], $N$-jetiness subtraction [44] and projection-to-born [45] have enabled a substantial number of new NNLO calculations of $(2 \rightarrow 2)$ processes at hadron colliders. All these calculations retain fully exclusive information on the final states, such that experimental data selections for fiducial cross sections can be realised, enabling a direct comparison to experimental data.

The $q_{T}$-subtraction method [41] uses the transverse momentum of the final state to separate off the infrared singular real radiation contributions. It can be applied to arbitrary colour-neutral final states, and enabled in the recent past in the calculation of NNLO corrections to vector boson pair production processes: $V \gamma$ [46], $Z Z$ [47, 48, 49], $W W$ [50, 51, 52], $W Z$ [53]. These processes provide in-depth tests of the coupling structure of the Standard Model and yield final state signatures that are relevant in many BSM searches. The inclusion of NNLO corrections to the total $W W$ cross section [50] and to related fiducial cross sections [51] largely explained an excess observed the LHC collaborations in Run 1, which had initially given rise to speculations of anomalous contributions or new physics effects, see Figure 3.

Top quark pair production is measured to a few per cent accuracy at the LHC, with more and more differential distributions becoming available. These data are now becoming input to various precision studies of the top quark mass, parton distributions and the strong coupling constant. NNLO corrections to the total cross section [54], to the forward-backward asymmetry [55] and to differential distributions [56] are computed, providing important input to these precision studies, see Figure 4. The large corrections to the forward-backward asymmetry [55] explained moreover an anomaly observed by the Fermilab Tevatron experiments.

QCD corrections to Higgs production in the vector boson fusion process can be related to corrections in deep inelastic scattering, which displays a closely related kinematics. Based on this 


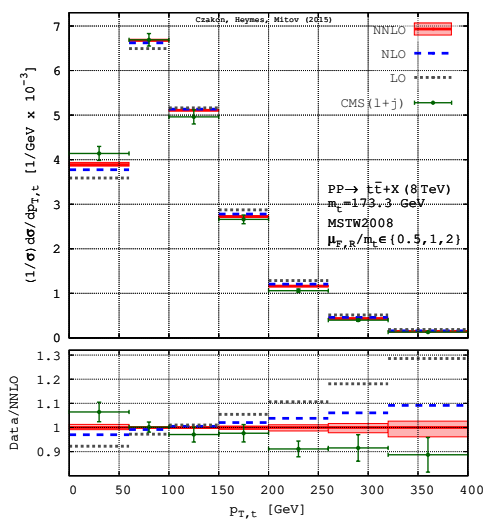

Figure 4: Transverse momentum distribution of top quarks to NNLO QCD. Figure from [56].
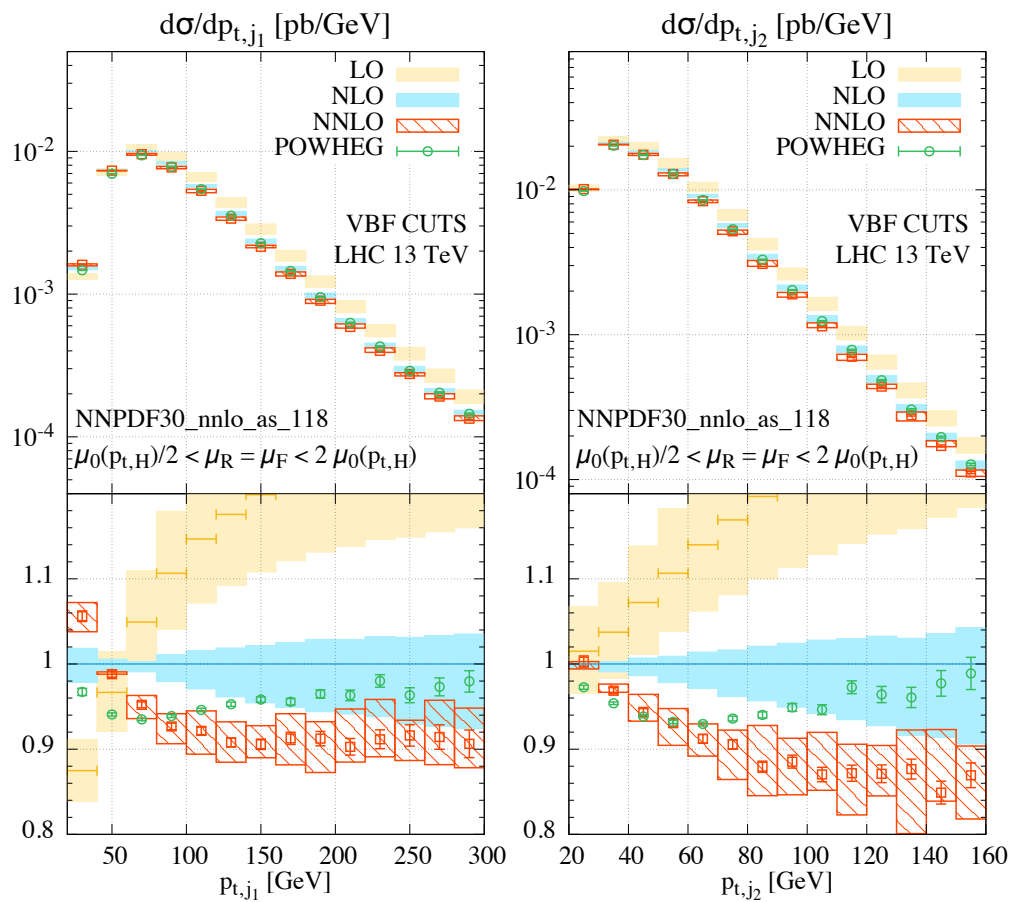

Figure 5: Leading and subleading jet distributions in vector boson fusion to NNLO QCD. Figure from [45].

relation, the NNLO [57] and most recently also N3LO corrections [58] to the inclusive vector boson fusion cross section could be derived, and were found to be rather small. A more differential treatment of this process, which was accomplished to NNLO in [45] reveals however that the kinematical distributions of the tagging jets are substantially modified through the higher order effects, see Figure 5, in a manner that is different from original parton shower expectations.

Three different calculations, each using a different method, of the NNLO corrections to Higgsplus-one-jet productions have become available [59, 60, 61, 62], indicating sizable corrections and stabilising the theoretical prediction. They include the decays to photon pairs [61, 62] and to four lepton final states [61], which allow for a direct comparison with the experimentally measured fidu- 

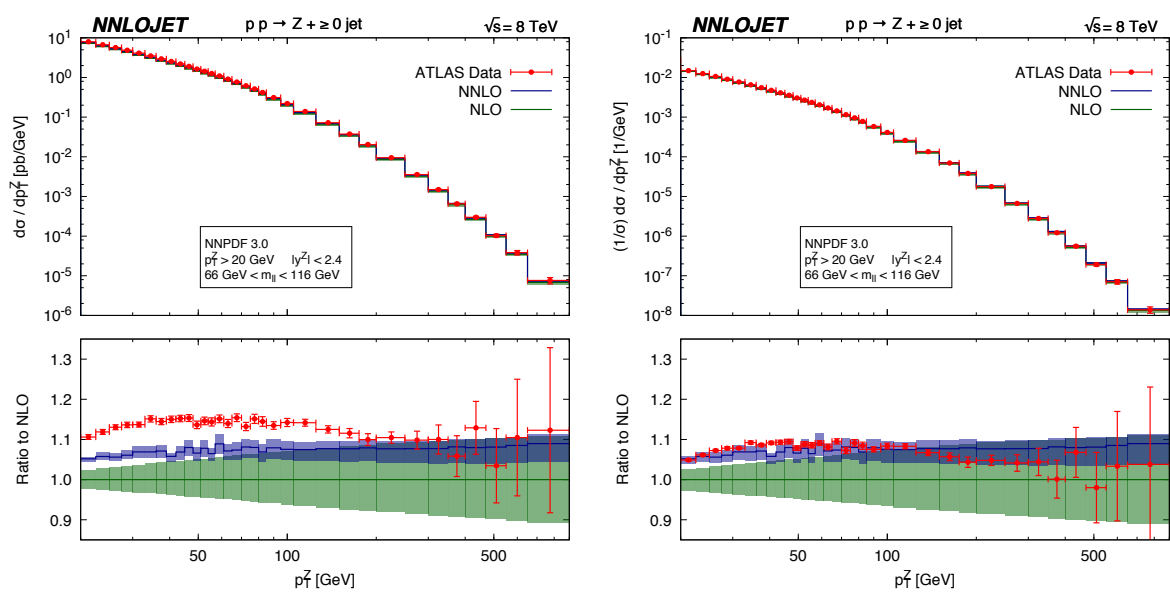

Figure 6: NNLO predictions for transverse momentum distribution of the $Z$ boson: absolute distribution (left) and normalized to total fiducial cross section (right). Figure from [66].

cial cross sections. With increasing luminosity, the Higgs boson transverse momentum distribution and Higgs-plus-one-jet cross sections will become precision observables, and the new calculations will allow a detailed investigation of Higgs properties and production dynamics.

Vector-boson-plus-jet production is routinely measured to high precision at the LHC, and can provide crucial input to global determinations of parton distributions. NNLO corrections to $W+$ $j[63]$ and $Z+j[64,65,66]$ were computed most recently. A comparison of the $Z$ boson transverse momentum distribution with data from ATLAS and CMS reveals that the shape of the data is described in an excellent way at NNLO, while a deviation in normalisation is observed, by the same amount as seen in the fiducial cross section for inclusive $Z$ production [66]. These findings call for a careful reinvestigation of the parametric ingredients that enter in the experimental analysis and the theoretical prediction, and demonstrate the physics potential of precision studies.

Inclusive jet production is the most basic QCD process; it is measured over a large kinematical range at the LHC, and precision data at lower energies are available for hadron-hadron collisions from the Tevatron and for lepton-hadron collisions from HERA. These data could become important ingredients to global determination of parton distributions, where they constrain especially the gluon distribution in the proton. A consistent treatment of these data in the context of NNLO parton distribution fits requires the NNLO corrections to inclusive jet and dijet production. For deep inelastic processes, these were computed most recently [67], Figure 7, and the calculation for hadron colliders is ongoing [68].

To turn the substantial number of recently accomplished NNLO calculations for hadron collider observables into tools for precision phenomenology, further developments are needed. At present, the typical NNLO codes require large supercomputer infrastructures to run upon, with typical runtimes of around 100'000 CPU-hours for a single set of parameter settings. To use these results in parameter determinations, a portable and fast parametrisation of the results, such as those provided at NLO by ApplGrid [69] or fastNLO [70] needs to be obtained.

To obtain NNLO-accurate predictions with full event information, it would be very much desirable to combine the fixed-order NNLO calculations with parton shower approaches. Based on 


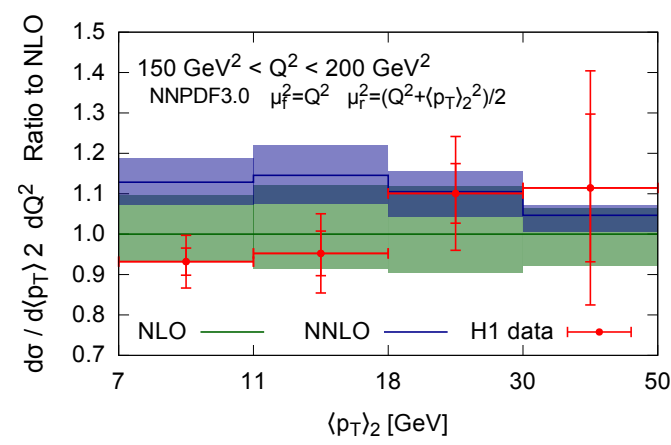

Figure 7: NNLO predictions for dijet production in deep inelastic scattering. Figure from [67].

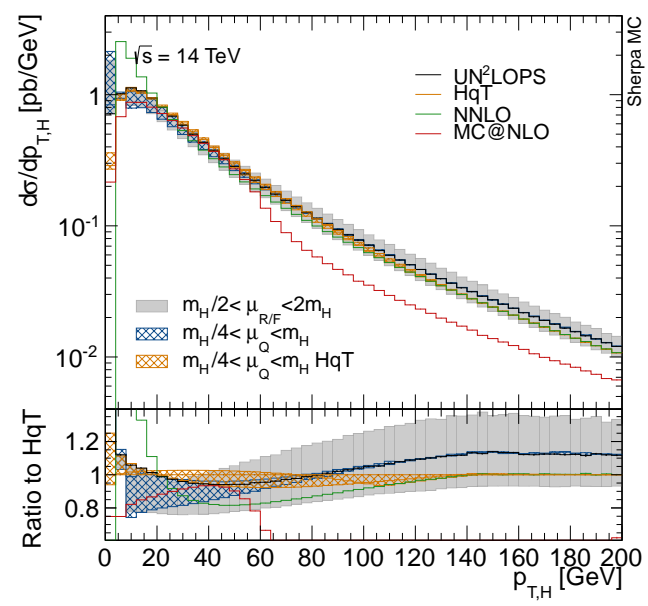

Figure 8: NNLO+PS predictions Higgs production. Figure from [73].

the NLO merging of different multiplicities, two methods have been developed: UN2LOPS [71] and NNLOPS [72]. They were applied to Higgs production [72, 73], Figure 8, and vector boson production [71, 74], as well as to $V H$ production [75].

\section{Multi-loop amplitudes and their applications}

Multi-loop amplitudes are a key ingredient to precision calculations of collider observables. Their calculation poses two fundamental challenges: the expression of the amplitude in terms of a small set of fundamental master integrals, and the evaluation of these master integrals. The former task can be accomplished with various reduction techniques (integration-by-parts, integrand reduction, unitarity methods), which are however not all fully developed beyond one loop, and which are getting increasingly complex in their application at higher orders and multiplicities.

The availability of all one-loop master integrals for arbitrary mass and momentum configurations was a major driver of the NLO revolution discussed above. Beyond one loop, master integrals 
are known only for specific processes, thereby limiting further progress on the calculation of corrections at NNLO and beyond. At present, two-loop corrections are limited to $(2 \rightarrow 2)$ amplitudes (with very recent advances towards integrals for $(2 \rightarrow 3)$ processes $[76,77,78]$ ), three-loop results are known for $(2 \rightarrow 1)$ amplitudes (form factors) and related single-scale quantities (coefficient functions, e.g. recently for heavy quark production in deep inelastic scattering [79]), while vacuum integrals and two-point functions are known to four loops, thereby enabling the calculation of total cross sections [80], $\beta$-functions [81] or magnetic moments [82].

Gluon fusion is the most important production mode for the Higgs boson at the LHC. This process receives very large corrections at NLO and NNLO. To obtain a reliable theoretical description of it, and to enable Higgs precision physics in the gluon fusion mode, consequently requires third order corrections. Owing to several technical advances in multi-loop calculations, this largescale project could be accomplished most recently [83], resulting in a small N3LO QCD correction. Combined with other types of corrections (quark mass effects, electroweak), a theoretical prediction of Higgs production in gluon fusion [84] that is accurate to $(+4.5 \%,-6.7 \%)$ can be obtained, thereby opening the path for precision studies of Higgs production in the upcoming LHC run.

\section{Outlook}

Precision calculations in QCD are crucial to ensure the optimal exploitation of LHC data in terms of Standard Model precision measurements and new physics searches.

During the past years, technological breakthroughs have enabled the calculation of NLO corrections to processes of arbitrary multiplicity and complexity. This NLO revolution has been enabled by a high degree of automation, and by a standardisation of interfaces between different contributions. NLO calculations are now routinely matched onto parton showers, and schemes for the combination of NLO results for processes of different multiplicity are emerging, thereby enabling NLO-accurate event samples for experimental analyses.

Very substantial process is being made on NNLO calculations for benchmark observables, with many new results appearing now. These calculations are currently being performed on a caseby-case basis. First steps are now being taken to match fixed-order NNLO calculations onto parton showers, and to combine them with analytical resummation techniques. Future developments in NNLO automation will have to address multiple challenges, especially in the calculation of twoloop integrals and amplitudes.

To obtain numerical predictions at NLO and NNLO, substantial computational resources are required. In order to enable the inclusion of these calculations in experimental studies, such as parameter extractions or parton distribution fits, more efficient forms for the distribution of the results (e.g. in the form of event files or coefficient functions) will have to be developed further, thereby requiring agreements on data structures and interfaces.

Very precisely measured inclusive cross sections demand predictions that are accurate even beyond NNLO. The recent calculation of the third-order corrections to inclusive Higgs production in gluon fusion proceeded alongside with important technical developments, which will enable future calculations at the same order for other precision observables. 


\section{References}

[1] T. Binoth et al., Comput. Phys. Commun. 181 (2010) 1612 [arXiv:1001.1307]; S. Alioli et al., Comput. Phys. Commun. 185 (2014) 560 [arXiv:1308.3462].

[2] C. F. Berger, Z. Bern, L. J. Dixon, F. Febres Cordero, D. Forde, H. Ita, D. A. Kosower and D. Maitre, Phys. Rev. D 78 (2008) 036003 [arXiv:0803.4180].

[3] G. Cullen et al., Eur. Phys. J. C 74 (2014) 3001 [arXiv:1404.7096].

[4] F. Cascioli, P. Maierhöfer and S. Pozzorini, Phys. Rev. Lett. 108 (2012) 111601 [arXiv:1111.5206].

[5] S. Badger, B. Biedermann, P. Uwer and V. Yundin, Comput. Phys. Commun. 184 (2013) 1981 [arXiv:1209.0100].

[6] V. Hirschi, R. Frederix, S. Frixione, M. V. Garzelli, F. Maltoni and R. Pittau, JHEP 1105 (2011) 044 [arXiv:1103.0621].

[7] G. Ossola, C. G. Papadopoulos and R. Pittau, JHEP 0803 (2008) 042 [arXiv:0711.3596].

[8] J. Bellm et al., Eur. Phys. J. C 76 (2016) 196 [arXiv:1512.01178].

[9] T. Gleisberg, S. Höche, F. Krauss, M. Schönherr, S. Schumann, F. Siegert and J. Winter, JHEP 0902 (2009) 007 [arXiv:0811.4622].

[10] J. Alwall et al., JHEP 1407 (2014) 079 [arXiv:1405.0301].

[11] J. M. Campbell, R. K. Ellis and C. Williams, JHEP 1107 (2011) 018 [arXiv:1105.0020].

[12] K. Arnold et al., Comput. Phys. Commun. 180 (2009) 1661 [arXiv:0811.4559].

[13] S. Badger, B. Biedermann, P. Uwer and V. Yundin, Phys. Rev. D 89 (2014) 034019 [arXiv:1309.6585].

[14] Z. Bern, L. J. Dixon, F. Febres Cordero, S. Höche, H. Ita, D. A. Kosower, D. Maitre and K. J. Ozeren, Phys. Rev. D 88 (2013) 014025 [arXiv:1304.1253].

[15] T. Melia, K. Melnikov, R. Rontsch and G. Zanderighi, Phys. Rev. D 83 (2011) 114043 [arXiv:1104.2327].

[16] F. Campanario, N. Kaiser and D. Zeppenfeld, Phys. Rev. D 89 (2014) 014009 [arXiv:1309.7259]; F. Campanario, M. Kerner, L. D. Ninh and D. Zeppenfeld, JHEP 1407 (2014) 148 [arXiv:1405.3972]; Eur. Phys. J. C 74 (2014) 3085 [arXiv:1407.7857].

[17] G. Bozzi, F. Campanario, V. Hankele and D. Zeppenfeld, Phys. Rev. D 81 (2010) 094030 [arXiv:0911.0438]; G. Bozzi, F. Campanario, M. Rauch and D. Zeppenfeld, Phys. Rev. D 84 (2011) 074028 [arXiv:1107.3149]; G. Bozzi, F. Campanario, M. Rauch, H. Rzehak and D. Zeppenfeld, Phys. Lett. B 696 (2011) 380 [arXiv:1011.2206].

[18] F. Campanario, T. M. Figy, S. Plätzer and M. Sjödahl, Phys. Rev. Lett. 111 (2013) no.21, 211802 [arXiv:1308.2932].

[19] N. Greiner, S. Höche, G. Luisoni, M. Schönherr, J. C. Winter and V. Yundin, JHEP 1601 (2016) 169 [arXiv:1506.01016].

[20] Z. Bern, L. J. Dixon, F. Febres Cordero, S. Höche, H. Ita, D. A. Kosower and D. Maitre, Comput. Phys. Commun. 185 (2014) 1443 [arXiv:1310.7439].

[21] S. Frixione and B. R. Webber, JHEP 0206 (2002) 029 [hep-ph/0204244].

[22] S. Frixione, P. Nason and C. Oleari, JHEP 0711 (2007) 070 [arXiv:0709.2092]. 
[23] S. Höche, F. Krauss, M. Schönherr and F. Siegert, JHEP 1304 (2013) 027 [arXiv:1207.5030].

[24] K. Hamilton, P. Nason and G. Zanderighi, JHEP 1210 (2012) 155 [arXiv:1206.3572].

[25] L. Lönnblad and S. Prestel, JHEP 1303 (2013) 166 doi:10.1007/JHEP03(2013)166 [arXiv:1211.7278 [hep-ph]].

[26] S. Kallweit, J. M. Lindert, P. Maierhöfer, S. Pozzorini and M. Schönherr, JHEP 1504 (2015) 012 [arXiv:1412.5157]; JHEP 1604 (2016) 021 [arXiv:1511.08692].

[27] S. Frixione, V. Hirschi, D. Pagani, H. S. Shao and M. Zaro, JHEP 1409 (2014) 065 [arXiv:1407.0823]; JHEP 1506 (2015) 184 [arXiv:1504.03446].

[28] S. Catani, L. Cieri, D. de Florian, G. Ferrera and M. Grazzini, Nucl. Phys. B 888 (2014) 75 [arXiv:1405.4827]; T. Ahmed, M. K. Mandal, N. Rana and V. Ravindran, Phys. Rev. Lett. 113 (2014) 212003 [arXiv:1404.6504].

[29] S. Catani, L. Cieri, D. de Florian, G. Ferrera and M. Grazzini, Nucl. Phys. B 881 (2014) 414 [arXiv:1311.1654]; M. Grazzini, S. Kallweit, D. Rathlev and M. Wiesemann, JHEP 1508 (2015) 154 [arXiv:1507.02565].

[30] Y. Li and H. X. Zhu, [arXiv:1604.01404].

[31] M. Dasgupta, F. Dreyer, G. P. Salam and G. Soyez, JHEP 1504 (2015) 039 [arXiv:1411.5182].

[32] A. Banfi, F. Caola, F. A. Dreyer, P. F. Monni, G. P. Salam, G. Zanderighi and F. Dulat, JHEP 1604 (2016) 049 [arXiv:1511.02886].

[33] T. Becher, M. Neubert, L. Rothen and D. Y. Shao, Phys. Rev. Lett. 116 (2016) 192001 [arXiv:1508.06645].

[34] T. Becher, X. Garcia i Tormo and J. Piclum, Phys. Rev. D 93 (2016) 054038 [arXiv:1512.00022].

[35] I. W. Stewart, F. J. Tackmann, J. Thaler, C. K. Vermilion and T. F. Wilkason, JHEP 1511 (2015) 072 [arXiv:1508.01516].

[36] I. W. Stewart, F. J. Tackmann and W. J. Waalewijn, Phys. Rev. Lett. 105 (2010) 092002 [arXiv:1004.2489].

[37] P. Pietrulewicz, F. J. Tackmann and W. J. Waalewijn, JHEP 1608 (2016) 002 [arXiv:1601.05088].

[38] Y. Li and H. X. Zhu, JHEP 1311 (2013) 080 [arXiv:1309.4391].

[39] J. R. Gaunt, M. Stahlhofen and F. J. Tackmann, JHEP 1404 (2014) 113 [arXiv:1401.5478]; JHEP 1408 (2014) 020 [arXiv:1405.1044]; T. Gehrmann, T. Lübbert and L. L. Yang, JHEP 1406 (2014) 155 [arXiv:1403.6451].

[40] T. Binoth and G. Heinrich, Nucl. Phys. B 585 (2000) 741 [hep-ph/0004013]; C. Anastasiou, K. Melnikov and F. Petriello, Phys. Rev. D 69 (2004) 076010 [hep-ph/0311311].

[41] S. Catani and M. Grazzini, Phys. Rev. Lett. 98 (2007) 222002 [hep-ph/0703012].

[42] M. Czakon, Phys. Lett. B 693 (2010) 259 [arXiv:1005.0274].

[43] A. Gehrmann-De Ridder, T. Gehrmann and E. W. N. Glover, JHEP 0509 (2005) 056 [hep-ph/0505111]; A. Daleo, T. Gehrmann and D. Maitre, JHEP 0704 (2007) 016 [hep-ph/0612257]; J. Currie, E. W. N. Glover and S. Wells, JHEP 1304 (2013) 066 [arXiv:1301.4693].

[44] R. Boughezal, X. Liu and F. Petriello, Phys. Rev. D 91 (2015) 094035 [arXiv:1504.02540]; J. Gaunt, M. Stahlhofen, F. J. Tackmann and J. R. Walsh, JHEP 1509 (2015) 058 [arXiv:1505.04794]. 
[45] M. Cacciari, F. A. Dreyer, A. Karlberg, G. P. Salam and G. Zanderighi, Phys. Rev. Lett. 115 (2015) 082002 [arXiv:1506.02660].

[46] M. Grazzini, S. Kallweit and D. Rathlev, JHEP 1507 (2015) 085 [arXiv:1504.01330].

[47] F. Cascioli et al., Phys. Lett. B 735 (2014) 311 [arXiv:1405.2219].

[48] M. Grazzini, S. Kallweit and D. Rathlev, Phys. Lett. B 750 (2015) 407 [arXiv:1507.06257].

[49] F. Caola, M. Dowling, K. Melnikov, R. Röntsch and L. Tancredi, JHEP 1607 (2016) 087 [arXiv:1605.04610].

[50] T. Gehrmann, M. Grazzini, S. Kallweit, P. Maierhöfer, A. von Manteuffel, S. Pozzorini, D. Rathlev and L. Tancredi, Phys. Rev. Lett. 113 (2014) 212001 [arXiv:1408.5243].

[51] M. Grazzini, S. Kallweit, S. Pozzorini, D. Rathlev and M. Wiesemann, JHEP 1608 (2016) 140 [arXiv:1605.02716].

[52] F. Caola, K. Melnikov, R. Röntsch and L. Tancredi, Phys. Lett. B 754 (2016) 275 [arXiv:1511.08617].

[53] M. Grazzini, S. Kallweit, D. Rathlev and M. Wiesemann, Phys. Lett. B 761 (2016) 179 [arXiv:1604.08576].

[54] M. Czakon, P. Fiedler and A. Mitov, Phys. Rev. Lett. 110 (2013) 252004 [arXiv:1303.6254].

[55] M. Czakon, P. Fiedler and A. Mitov, Phys. Rev. Lett. 115 (2015) 052001 [arXiv:1411.3007].

[56] M. Czakon, D. Heymes and A. Mitov, Phys. Rev. Lett. 116 (2016) 082003 [arXiv:1511.00549]; [arXiv:1606.03350]; M. Czakon, P. Fiedler, D. Heymes and A. Mitov, JHEP 1605 (2016) 034 [arXiv:1601.05375].

[57] P. Bolzoni, F. Maltoni, S. O. Moch and M. Zaro, Phys. Rev. Lett. 105 (2010) 011801 [arXiv:1003.4451].

[58] F. A. Dreyer and A. Karlberg, Phys. Rev. Lett. 117 (2016) 072001 [arXiv:1606.00840].

[59] R. Boughezal, F. Caola, K. Melnikov, F. Petriello and M. Schulze, Phys. Rev. Lett. 115 (2015) 082003 [arXiv:1504.07922].

[60] R. Boughezal, C. Focke, W. Giele, X. Liu and F. Petriello, Phys. Lett. B 748 (2015) 5 [arXiv:1505.03893].

[61] F. Caola, K. Melnikov and M. Schulze, Phys. Rev. D 92 (2015) 074032 [arXiv:1508.02684].

[62] X. Chen, J. Cruz-Martinez, T. Gehrmann, E. W. N. Glover and M. Jaquier, [arXiv:1607.08817].

[63] R. Boughezal, C. Focke, X. Liu and F. Petriello, Phys. Rev. Lett. 115 (2015) 062002 [arXiv:1504.02131].

[64] R. Boughezal, J. M. Campbell, R. K. Ellis, C. Focke, W. T. Giele, X. Liu and F. Petriello, Phys. Rev. Lett. 116 (2016) 152001 [arXiv:1512.01291]; R. Boughezal, X. Liu and F. Petriello, Phys. Lett. B 760 (2016) 6 [arXiv:1602.05612].

[65] A. Gehrmann-De Ridder, T. Gehrmann, E. W. N. Glover, A. Huss and T. A. Morgan, Phys. Rev. Lett. 117 (2016) 022001 [arXiv:1507.02850].

[66] A. Gehrmann-De Ridder, T. Gehrmann, E. W. N. Glover, A. Huss and T. A. Morgan, JHEP 1607 (2016) 133 [arXiv:1605.04295].

[67] J. Currie, T. Gehrmann and J. Niehues, Phys. Rev. Lett. 117 (2016) 042001 [arXiv:1606.03991]. 
[68] A. Gehrmann-De Ridder, T. Gehrmann, E. W. N. Glover and J. Pires, Phys. Rev. Lett. 110 (2013) 162003 [arXiv:1301.7310]; J. Currie, A. Gehrmann-De Ridder, E. W. N. Glover and J. Pires, JHEP 1401 (2014) 110 [arXiv:1310.3993].

[69] T. Carli, D. Clements, A. Cooper-Sarkar, C. Gwenlan, G. P. Salam, F. Siegert, P. Starovoitov and M. Sutton, Eur. Phys. J. C 66 (2010) 503 [arXiv:0911.2985].

[70] D. Britzger et al. [fastNLO Collaboration], Proceedings of DIS2012, p.217 [arXiv:1208.3641].

[71] S. Höche, Y. Li and S. Prestel, Phys. Rev. D 91 (2015) 074015 [arXiv:1405.3607].

[72] K. Hamilton, P. Nason, E. Re and G. Zanderighi, JHEP 1310 (2013) 222 [arXiv:1309.0017].

[73] S. Höche, Y. Li and S. Prestel, Phys. Rev. D 90 (2014) no.5, 054011 [arXiv:1407.3773].

[74] A. Karlberg, E. Re and G. Zanderighi, JHEP 1409 (2014) 134 [arXiv:1407.2940].

[75] W. Astill, W. Bizon, E. Re and G. Zanderighi, JHEP 1606 (2016) 154 [arXiv:1603.01620].

[76] S. Badger, H. Frellesvig and Y. Zhang, JHEP 1312 (2013) 045 [arXiv:1310.1051]; S. Badger, G. Mogull, A. Ochirov and D. O’Connell, JHEP 1510 (2015) 064 [arXiv:1507.08797].

[77] T. Gehrmann, J. M. Henn and N. A. Lo Presti, Phys. Rev. Lett. 116 (2016) 062001 [arXiv:1511.05409].

[78] C. G. Papadopoulos, D. Tommasini and C. Wever, JHEP 1604 (2016) 078 [arXiv:1511.09404].

[79] J. Ablinger, A. Behring, J. Blümlein, A. De Freitas, A. von Manteuffel and C. Schneider, Nucl. Phys. B 890 (2014) 48 [arXiv:1409.1135]; A. Behring, J. Blümlein, A. De Freitas, A. Hasselhuhn, A. von Manteuffel and C. Schneider, Phys. Rev. D 92 (2015) 114005 [arXiv:1508.01449].

[80] P. A. Baikov, K. G. Chetyrkin and J. H. Kühn, Phys. Rev. Lett. 101 (2008) 012002 [arXiv:0801.1821]; P. A. Baikov, K. G. Chetyrkin, J. H. Kühn and J. Rittinger, Phys. Rev. Lett. 108 (2012) 222003 [arXiv:1201.5804].

[81] P. A. Baikov, K. G. Chetyrkin and J. H. Kühn, [arXiv:1606.08659]; T. Luthe, A. Maier, P. Marquard and Y. Schröder, JHEP 1607 (2016) 127 [arXiv:1606.08662].

[82] A. Kurz, T. Liu, P. Marquard, A. V. Smirnov, V. A. Smirnov and M. Steinhauser, Phys. Rev. D 92 (2015) 073019 [arXiv:1508.00901]; Phys. Rev. D 93 (2016) 053017 [arXiv:1602.02785].

[83] C. Anastasiou, C. Duhr, F. Dulat, F. Herzog and B. Mistlberger, Phys. Rev. Lett. 114 (2015) 212001 [arXiv:1503.06056].

[84] C. Anastasiou, C. Duhr, F. Dulat, E. Furlan, T. Gehrmann, F. Herzog, A. Lazopoulos and B. Mistlberger, JHEP 1605 (2016) 058 [arXiv:1602.00695]. 